\title{
Schreibtischverlängerung dank Access-Control-System von HIN
}

Das Kantonsspital Luzern beschreitet neue Wege der Zusammenarbeit. Zuweisende oder nachbehandelnde Ärzte erhalten von extern neu direkten Zugriff auf klinische Daten des Spitals. Damit wird die Kontinuität im Behandlungsprozess der Patienten deutlich verbessert. HIN ist verantwortlich für die sichere Anbindung der externen Ärzte an das Spital.

Andreas Nievergelt, Leiter Einzelkunden, HIN Health Info Net

Korrespondenz: Health Info Net AG Pflanzschulstrasse 3 CH-8411 Winterthur Tel. 0522350270 Fax 0522350272 info@hin.ch

\section{Ausgangslage}

Das Kantonsspital Luzern (KSL) ist mit 679 Betten das grösste Spital der Innerschweiz. 435 Ärzte und 2000 Pflegende betreuten im Jahr 2005 rund 40000 stationäre Patienten und Notfälle. Während des Spitalaufenthalts eines einzelnen $\mathrm{Pa}$ tienten entstehen je nach Fall die unterschiedlichsten Daten. Jährlich sind dies über 300000 Dokumente und Bilder, vom Arztbericht über Laborbefunde bis hin zu Computertomogrammen (CT), Magnetresonanz- (MR) und konventionellen Röntgenaufnahmen (CR).

Um dieser Datenmenge Herr zu werden, führte das KSL die verschiedenen Datenbanken in einem mehrjährigen IT-Projekt zusammen. Das Data-Management-System «i-engine» dient dabei als Dach über den verschiedenen Datenbanken. Heute lagern bereits 2,3 Millionen Dokumente und Bilder in der elektronischen Dokumentenverwaltung. Ein Spitalarzt kann die gesammelten Berichte und Bilder eines einzelnen Patienten dadurch in Sekundenschnelle auf den Bildschirm holen. Mit einem Mausklick öffnet er die einzelnen Befunde und kann sich so ein Bild über den Patienten machen.

\section{Aufgabenstellung}

Diese elektronische Dokumentenverwaltung stösst bei den Anwendern im KSL auf sehr breite Akzeptanz. «Das genügte uns aber noch nicht», erklärt Informatikleiter Peter Steinmann. «Für das KSL als Zentrumsspital ist eine gute Zusammenarbeit mit Ärzten ausserhalb des Spitals sehr wichtig. Deshalb haben wir uns entschlossen, unser System für diese Benutzergruppe zu öffnen.» Für die Umsetzung waren zwei Bedingungen zentral:

1. Die Datensicherheit muss zu jeder Zeit gewahrt bleiben.
2. Es muss immer nachvollziehbar sein, welcher Spitalarzt welchem Hausarzt oder Spezialisten Zugriff auf welche Daten gewährt.

Das System sollte einfach und benutzerfreundlich sein. Um die Spitalinformatik nicht mit neuen Aufgaben zu belasten, sollte zudem ein hoher Automatisierungsgrad erreicht werden. Mit diesen Vorgaben wurde im Dezember 2004 der Entscheid gefällt, das Projekt in Angriff zu nehmen.

\section{Lösung}

Heute ist das Projekt umgesetzt. 200 Hausärzte und Spezialisten aus der Innerschweiz können beim KSL Informationen über ihre dort behandelten Patienten abholen. Die Resonanz der externen Ärzte ist sehr gut. Sie staunen über die Schnelligkeit und Benutzerfreundlichkeit des Systems und sind von der Qualität der Berichte und Bilder beeindruckt. Informatikleiter Peter Steinmann: «Ich rechne bereits im ersten Jahr mit über 10000 Zugriffen.»

Und so funktioniert die Zusammenarbeit: Wünscht ein Hausarzt Einsicht in die Spitalunterlagen eines Patienten, so stellt er eine Anfrage an den zuständigen Spitalarzt. Dieser kann die Unterlagen mit wenigen Mausklicks freischalten. Im Systemlog wird gleichzeitig vermerkt, wer wem die Zugriffsberechtigung auf welche Dokumente erteilt hat. Der Hausarzt erhält über HIN ein verschlüsseltes E-Mail, das den Internetlink auf die Daten seines Patienten enthält. Mit nur zwei Mausklicks hat sich der Arzt in das System eingeloggt. Weil er über HIN bereits authentifiziert wurde, ist keine Passworteingabe nötig. Per Mausklick kann der Hausarzt sodann Laborbefunde, Arztberichte, CRs, CTs und MRs abrufen. Letztere sind im JEPG-Format 


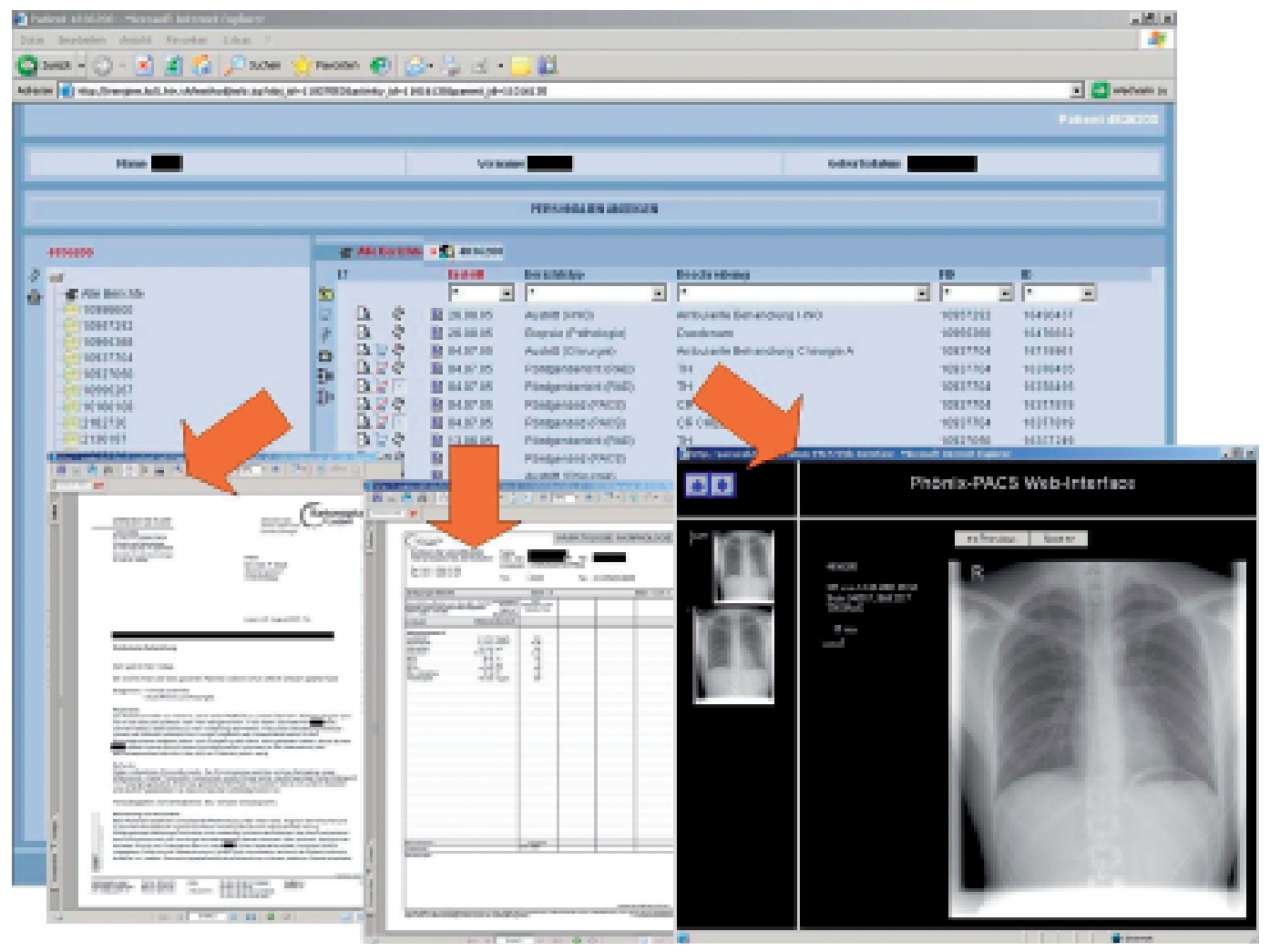

von ihrer Qualität her in den meisten Fällen genügend. «Wir haben das System von Anfang an so konzipiert, dass es für externe Ärzte möglichst einfach zu bedienen ist. Mit dem direkten Zugriff über HIN und dem übersichtlichen Arbeitsbereich ist uns dies optimal gelungen», urteilt Steinmann. Der Arzt kann dabei nur Daten einsehen, für die er freigeschaltet wurde. Nach 30 Tagen wird der Zugriff automatisch wieder gesperrt.

Damit der externe Arzt das System nutzen kann, müssen zwei Voraussetzungen erfüllt sein: Er braucht einen Breitbandinternetzugang (emp-

\section{Access Control Service (ACS)}

Und so funktioniert's: Für den geschützten Zugriff auf eine geschützte Ressource - sei dies ein Server, eine Datenbank oder eine Applikation - wird die HIN-Sicherheitstechnologie eingesetzt. Anstatt mit einem Benutzernamen und Passwort erfolgt die Authentifikation auf Basis von X.509-Zertifikaten. Über eine sichere Internetverbindung (SSL-Verbindung mit beidseitiger Authentifikation) gelangen die Abonnenten von HIN zuerst zum HIN-Rechenzentrum. Dort wird überprüft, ob der Benutzer für den Zugriff auf die Zielapplikation berechtigt wurde. Ist dies der Fall, wird der Benutzer mit der gewünschten Applikation verbunden. Das Login kann dort automatisiert erfolgen, das heisst, es ist keine Passworteingabe mehr nötig. Damit sind die HIN Access Control Services nicht nur sicherer, sondern auch bequemer als manche Online-Banking-Systeme. fohlen sind mindestens $1000 \mathrm{kbps}$ Downstream) und ein HIN-Abo. Damit kann er über eine sichere Internetverbindung auf die gewünschten Informationen zugreifen. In der Arztpraxis steht ein einzelner Untersuchungsbericht oder auch ein vollständiges Patientendossier in Sekundenschnelle zur Verfügung.

\section{Fazit}

Indem das KSL externen Ärzten einen Zugriff auf die medizinischen Informationen des Spitals ermöglicht, geht es neue Wege in der Einbindung der niedergelassenen Ärzte in den medizinischen Behandlungsprozess. Die Wirksamkeit der eigenen IT-Systeme wird dadurch potenziert. Mit dem Anschluss an die HIN-E-Health-Plattform delegierte das KSL die Sicherheitsfrage an HIN. Die spitaleigenen IT-Spezialisten brauchen sich dadurch nicht um Entwicklung, Roll-Out und Support einer eigenen Sicherheitslösung $\mathrm{zu}$ kümmern. Steinmann: «Ohne HIN hätten wir den Zugriff von aussen auf die Patientendaten gar nicht umsetzen können.»

Die Ärzte im KSL kennen die Oberfläche der i-engine bereits und schätzen die schnelle Übersicht. Da auch sie unter permanentem Zeitdruck stehen, haben sie das schnelle Tool bereits in ihren Arbeitsalltag integriert. Durch die effiziente elektronische Handhabung spart das 
Spitalpersonal deutlich an Zeit. Der Hausarzt wiederum hat die gewünschten Dokumente bereits, bevor der Patient bei ihm erscheint und nicht erst Tage später. Und die Kosten? Die entstehenden Kosten könnten laut KSL im Vollbetrieb allein durch die eingesparten Portospesen gedeckt werden.

Zur Information: An der eHealthCare 2006 referieren Stefan Müller (HIN) und Peter Steinmann (Informatikleiter KSL) über die durch HIN gewährleistete Datensicherheit und das Informationssystem des KSL.

\section{Kommentar: «Das ist E-Health - pragmatisch und effizient!»}

\section{Philip Baumann, VR-Delegierter, HIN Health Info Net}

E-Health ist (leider) längst eine Projektionsfläche für alle möglichen und unmöglichen Innovationen im Gesundheitswesen geworden. Dabei wäre E-Health etwas sehr Konkretes.

Viele Ärztinnen und Ärzte arbeiten bereits mit E-Health, ohne dass ihnen dies bewusst ist. Das wäre ja auch das Ziel: E-Health darf nicht zu komplexeren und aufwendigeren Lösungen führen. Die aufgezeigte Lösung im Kantonsspital Luzern zeigt, dass dies heute möglich ist!

So wird über HIN der Informationsfluss entlang dem Behandlungspfad des Patienten verbessert und beschleunigt. Andere Ärztinnen schicken ihren Kollegen ein verschlüsseltes E-Mail, um einen Patienten für eine Untersuchung anzumelden. Oder holen Laborergebnisse direkt auf dem Server des Labors ab, um sie in die eigene Krankengeschichte zu integrieren. Damit ist auch klar, was E-Health ist und sein muss: eine Vereinfachung, Beschleunigung und Erleichterung der medizinischen und der administrativen Prozesse im Alltag. Oder würden Sie E-Mails verschicken, wenn dies umständlicher, langsamer und teurer wäre als ein Brief?

HIN geht deshalb einen einfachen und pragmatischen Weg, um die mit E-Health angestrebten, wichtigen Prozessinnovationen zu realisieren. Das grosse Potential von E-Health lässt sich nur in kleinen, pragmatischen Schritten erschliessen, die sich für alle Beteiligten lohnen.

\section{Schweizerischer eHealthcare-Kongress}

Konferenz \& Fachausstellung, 28./29. September 2006, GZI Forschungszentrum, Nottwil/LU

Der Kongress richtet sich an alle ICT- und Health Professionals der Schweiz und ist Fachmesse und Konferenz zugleich. eHealthCare.ch vereinigt über 1500 führende Health Professionals und ICT-Entscheidungsträger aus dem Spitalsektor, dem ambulanten Sektor, der Industrie, dem Versicherungswesen und der gesamten Gesundheitsbranche.

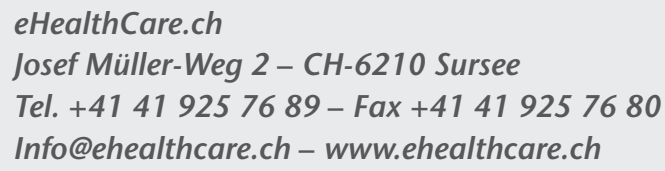

